\title{
The Physical Dose Difference of Three Types of Implantation on the CTV and Lungs in Interstitial Brachytherapy
}

\author{
Jing Zhang, Bo Yang, Haowen Pang, Guangpeng Zhang, Renjin Chen, Lei Li
}

Department of Oncology, The Affiliated Hospital of Southwest Medical University, Luzhou, China

Email address:

yangbo_lz@163.com (Bo Yang)

\section{To cite this article:}

Jing Zhang, Bo Yang, Haowen Pang, Guangpeng Zhang, Renjin Chen, Lei Li. The Physical Dose Difference of Three Types of Implantation on the CTV and Lungs in Interstitial Brachytherapy. Science Journal of Public Health. Vol. 8, No. 2, 2020, pp. 50-55.

doi: $10.11648 /$ j.sjph.20200802.14

Received: April 11, 2020; Accepted: May 3, 2020; Published: May 28, 2020

\begin{abstract}
Purpose: To study the difference of physical dose of target volume and lungs among actual irregular arrangement multi-needle interstitial brachytherapy plan (AIBP), virtual regular arrangement multi-needle interstitial brachytherapy plan (VRBP) and virtual single needle center point interstitial brachytherapy plan (VSBP). Methods: According to the inclusion criteria: the CTV shape was approximately spherical and multiply needles arrangement was irregular. Thirteen lung cancer patients underwent interstitial brachytherapy were collected. Based on the thirteen CT data sets, the AIBP, VRBP and VSBP were respectively designed, then we collected the dose metrics involving: the minimum dosage received by $95 \%$ of the CTV (D95), D90, D80, D50 and D30; the percentage of lung volume receiving a dose of $\geq 5$ Gy (V5), V20, V30 and the mean lung dose (MLD); D50 of heart; the maximum dose (Dmax) of spinal cord and the Dmax of ribs. The differences were tested by the two-sample paired (Wilcoxon) signed rank test, a $P$ value less than 0.05 was considered statistically significant. Result: The differences of D95, D90, D80, D50 and D30 of CTV, D50 of heart, Dmax of spinal and Dmax of ribs were not statistical significant $(P>0.05)$ and the V5, V20, V30 and MLD of lungs and ipsilateral lung were statistical significant $(P<0.05)$ between AIBP and VRBP. The differences of D95, D90, D80 and D50 of CTV, D50 of heart, Dmax of spinal cord, Dmax of ribs, V5, V20, V30 and MLD of lungs and ipsilateral lung were not statistical significant $(P>0.05)$ except for D30 of CTV $(P<0.05)$ between AIBP and VRBP. Conclusion: In interstitial brachytherapy for lung tumor, the arrangement of implantation needles could influenced the dose distribution in target and lungs. If the CTV shape could be approximately considered to be a spherical and a regular arrangement of multiply needles was difficult to achieve; the lung dose of the AIBP might have no obvious advantage over the VSBP and the VSBP should be worth a try.
\end{abstract}

Keywords: Interstitial Brachytherapy, Lung Cancer, Dose Distribution

\section{Introduction}

A basic principle of radiotherapy planning is to maximize the target dose, as much as possible to reduce the amount of normal tissue [1]. In interstitial radiotherapy, in order to meet this principle, a parallel arrangement of the multiple implantation needles with uniformly-spaced was recommend [2].

However, if the tumor was located in the chest and surrounded by ribs, the route of implantation needles were greatly restricted. Then, it becomes difficult to achieve a regular arrangement of multiple implantation needles. Thus, in most of clinical cases, multiple implantation needles arrangement was non parallel nor uniformly-spaced (Figure $1 \mathrm{~g})$. This might lead to an increasing of lung dose comparing with the regular arrangement of multiple implantation needles.

In order to quantitatively analyze the effect of the arrangement of multiple implantation needles on the dose distribution and seek an alternative method to take place of the irregular arrangement multiple implantation needles interstitial radiotherapy, we designed this experiment. 


\section{Materials and Methods}

\subsection{Patients Materials and Equipment}

We collected thirteen CT data sets of lung cancer patients underwent interstitial brachytherapy by a iridium 192 sources afterloading unit (microSelectron-HDR, Elekta, Netherlands) between March 2013 and December 2016 (Table 1). All CT images were obtained using a CT machine (lightspeed plus 4, General electric company, USA) with a thickness of $5 \mathrm{~mm}, 120 \mathrm{kV}$ and $200 \mathrm{~mA}$. The interstitial brachytherapy plans were programmed in three dimensional (3D) radiotherapy planning system ( Oncentra 4.3, Elekta, Sweden).

In each CT data set, at least two needles with course intersection had been implanted form the available ribs' interval. All of the thirteen CTV shape could be approximately considered to be a spherical. If we defined the maximum diameter of CTV as the " $a$ " and the minimum diameter as the " $b$ " in the maximum CTV CT image; the value of the number of CT slices containing CTV multiplied by $5 \mathrm{~mm}$ was defined as the "c". Then, all the thirteen CTV sizes met the criteria: $a<1.5 b, a<1.5 c, b<1.5 a, b<1.5 c$, $c<1.5 a, c<1.5 b$.

Table 1. Patient characteristics.

\begin{tabular}{ll}
\hline Characteristic & Frequency (n)/ Median (range) \\
\hline Total no. of patients (n) & 13 \\
Gender & \\
Males & 8 \\
Females & 5 \\
Location (n) & \\
Left lung & 9 \\
Right lung & 4 \\
No. of needles & $3(2-5)$ \\
CTV (cm3) & $30.33(6.90-150.13)$ \\
Lungs (cm3) & $2579.00(2341.00-2995.25)$ \\
Ipsilateral lung (cm3) & $1188.50(1067.00-1507.75)$ \\
Spinal cord $(\mathrm{cm} 3)$ & $22.47(18.12-25.00)$ \\
Heart (cm3) & $477.50(376.75-604.25)$ \\
Ribs (cm3) & $19.25(12.41-27.51)$ \\
\hline
\end{tabular}

CTV: Clinical target volume.

\subsection{Actual Irregular Arrangement Multi-needle Interstitial Brachytherapy Plan (AIBP)}

AIBP: the actual implantation needles were adopted for planning, all the dwell points were located in the actual needles and assure a location at $5 \mathrm{~mm}$ below the CTV surface using source dwell positions at a distance of $2.5-7.5 \mathrm{~mm}$ (Figure 1). 120 Gy was adopted as the prescription dose and the graphical optimization tool was used; plans were accepted if the following constrains were met:

The the minimum dosage received by $95 \%$ of the CTV (D95) ranged between 119.50Gy and 120.50Gy; meanwhile, the percentage of lung volume received 20Gy (V20) was as low as possible (in most casd, no more than $20 \%$ were adopted as a constrain).
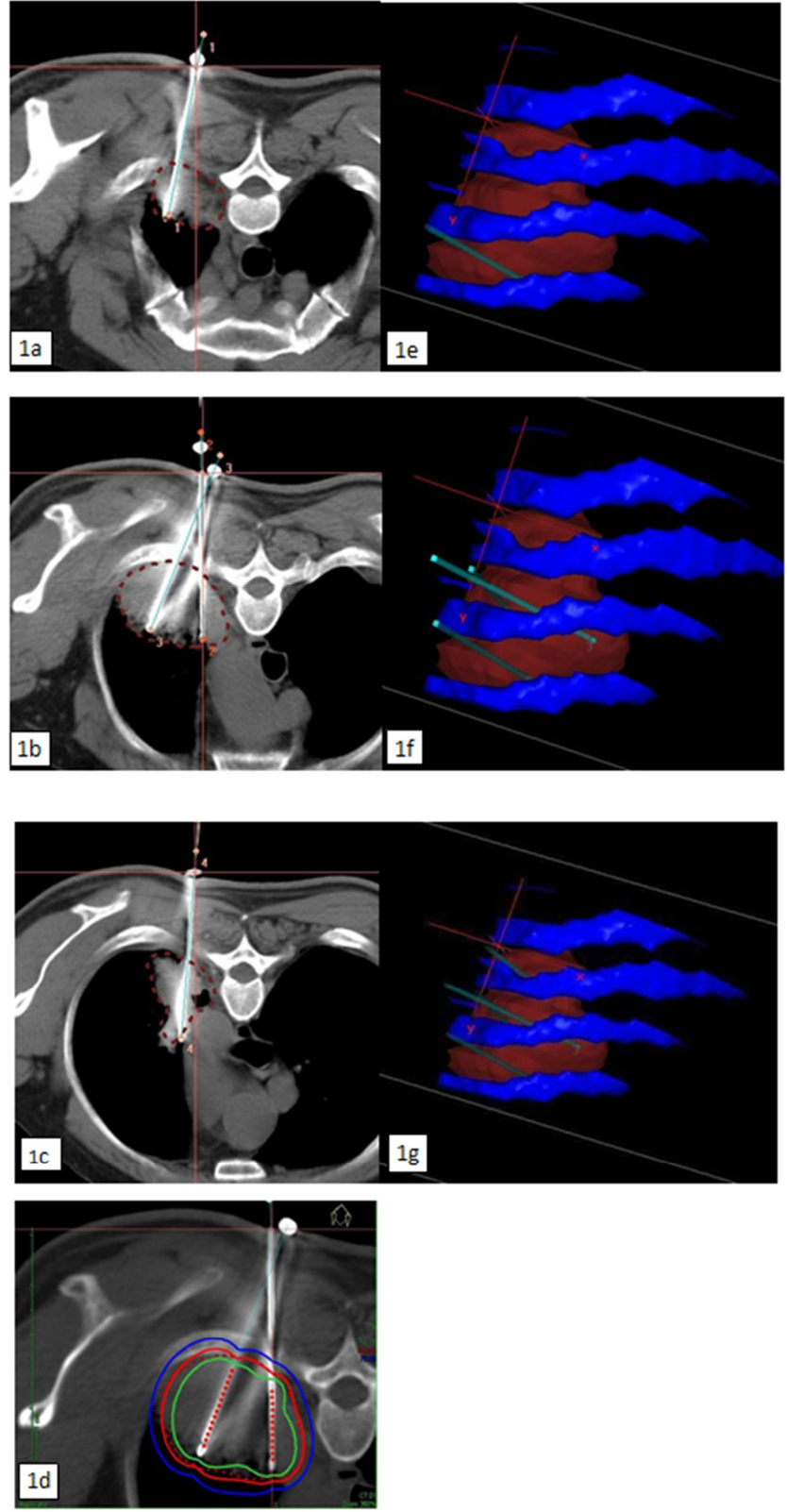

Figure 1. Schematic diagram of AIBP In this case, a total of 4 needles were inserted form the ribs' interval. 1a-1c: the CT images of actual implantation needles; 1e-1g: 3D image, bule structure were ribs, red structure was CTV, sky-bule structure were the implantation needles; 1d: dose distribution of AIBP in CT image, the red dots were dwell points, the red dotted line was CTV contour, the solid lines were isodose curves (the blue solid lines was $70 \%$ of prescription isodose curves, the red solid line was $100 \%$ of prescription isodose curves, the green solid line was $150 \%$ of prescription isodose curves).

\subsection{Virtual Regular Arrangement Multi-needle Interstitial Brachytherapy Plan (VRBP)}

VRBP: for a purpose of regular arrangement of multiply needles, hypothetical implantation needles were designed and all dwell points were located in the hypothetical needles. Those hypothetical needles were parallel with spacing of 1-1.5 $\mathrm{cm}$, some hyopthetical needles might pass through the ribs (Figure 2). The median number of needles was 7 (range, 3-11). Plans were optimized and accepted by the same constrains used in AIBP. 


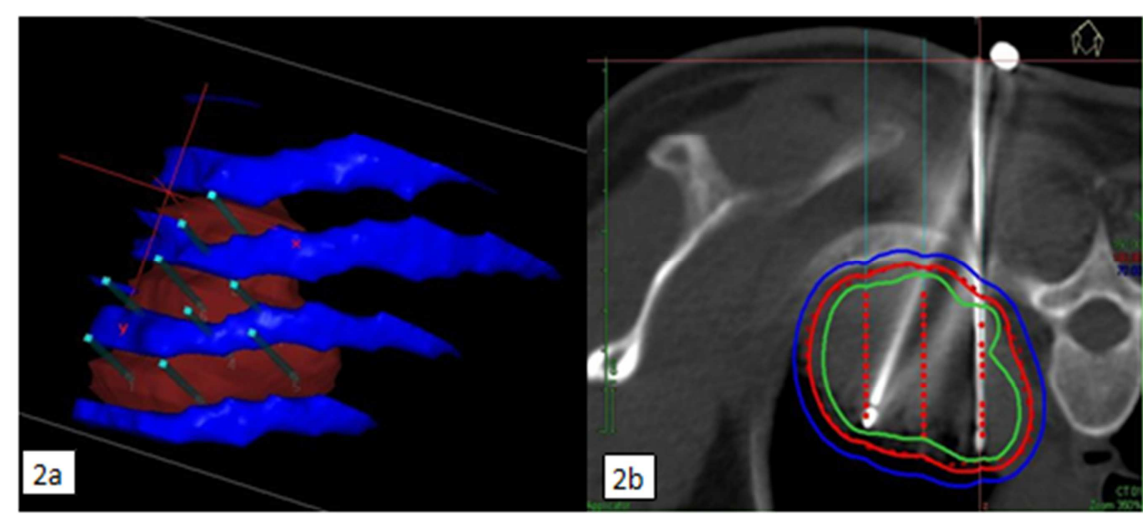

Figure 2. Schematic diagram of VRBP In this case, a total of 9 needles were designed passing through the ribs, needles were parallel with spacing of 1-1.5mm.

\subsection{Virtual Single Needle Center Point Interstitial Brachytherapy Plan (VSBP)}

VSBP: one hypothetical implantation needle was designed entrying form the ribs' interval. The hypothetical implantation needle passed through the center of the CTV and one dwell point was located at the CTV center (Figure 3). Plans were optimized and accepted by the same constrains used in AIBP.

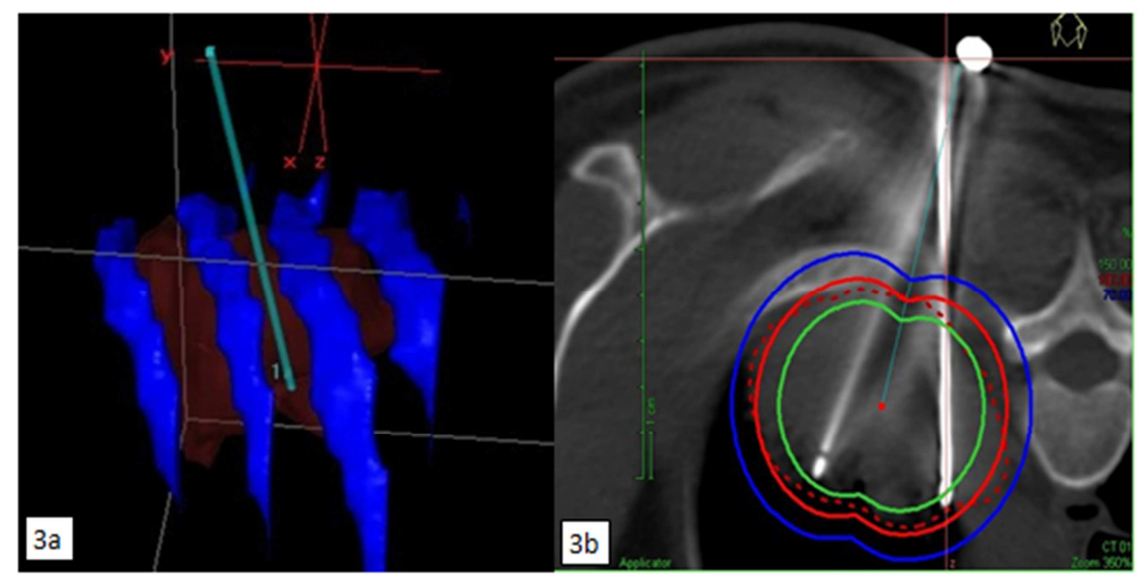

Figure 3. Schematic diagram of VSBP In this case, one hypothetical implantation needle was designed entrying form the ribs' interval and one dwell point was located at the CTV center.

\subsection{Dose Metrics and Statistical Analyses}

Dose metrics including: D95, D90, D80, D50 and D30 of CTV, V5, V20, V30 and the mean lung dose (MLD) for lungs and ipsilateral lung, D50 of heart, the maximum dose (Dmax) of spinal cord and the Dmax of ribs. The dose metrics data were tested for statistical significant for AIPB vs VRBP and AIPB vs VIBP. The two-sample paired signed rank Wilcoxon test was used. A $P$ value less than 0.05 was considered statistically significant.

\section{Results}

The differences of D95, D90, D80, D50, D30, D50 of heart, Dmax of spinal cord and Dmax of ribs were not statistical significant $(P>0.05)$, and the V5, V20, V30 and MLD were statistical significant $(P<0.05)$ between AIBP and VRBP (Table 2). All the dose metrics expect for D30 were not statistical significant $(P>0.05)$ between AIBP and VSBP (Table 3).

Table 2. Dose metrics between VRBP and AIPB.

\begin{tabular}{llll}
\hline & VRBP & AIPB \\
\hline & $D 95$ & $120.11(120.03-120.35)$ & $120.18(119.77-120.49)$ \\
Target dose (Gy) & $D 90$ & $132.59(131.92-138.80)$ & $138.05(135.72-139.78)$ \\
& $D 80$ & $158.39(153.63-172.97)$ & $165.12(160.02-177.79)$ \\
& $D 50$ & $252.57(234.18-311.07)$ & $262.33(245.89-319.85)$ \\
& $D 30$ & $347.30(319.47-389.61)$ & $361.06(339.73-400.54)$ \\
All lung (\%) & $V 5$ & $29.48(18.42-54.72)$ & $43.04(29.54-63.09)$ \\
& $V 20$ & $9.02(3.96-18.44)$ & $11.03(8.29-21.48)$ \\
& $V 30$ & $4.31(2.53-14.61)$ & $7.69(5.30-15.10)$ \\
\end{tabular}




\begin{tabular}{lllll}
\hline & & VRBP & AIPB & P \\
\hline & V5 & $63.64(47.18-85.05)$ & $70.63(59.86-91.70)$ & $<0.01$ \\
Ipsilateral lung (\%) & V20 & $17.74(12.69-42.72)$ & $23.91(17.09-47.94)$ & $<0.01$ \\
& V30 & $8.84(7.01-24.28)$ & $13.04(11.78-29.55)$ & $<0.01$ \\
Heart (Gy) & MLD & $13.76(7.73-20.89)$ & $16.91(14.24-29.71)$ & $<0.01$ \\
Spinal cord (Gy) & D50 & $3.03(2.77-7.15)$ & $3.63(3.08-8.17)$ & 0.77 \\
Ribs (Gy) & Dmax & $18.72(7.48-43.16)$ & $20.31(8.35-52.96)$ & 0.64 \\
\end{tabular}

VRBP: virtual regular arrangement multi-needle interstitial brachytherapy plan; AIPB: actual irregular arrangement multi-needle interstitial brachytherapy plan; Dx: the the minimum dosage received by $\mathrm{x} \%$ of the CTV; Vx: the percentage of lung volume received $\mathrm{x}$ Gy; MLD: the mean lung dose.

Table 3. Dose metrics between VSBP and AIPB.

\begin{tabular}{llll}
\hline & & VSBP & AIPB \\
\hline & $D 95$ & $120.34(120.04-120.46)$ & $120.18(119.77-120.49)$ \\
& $D 90$ & $139.59(134.92-142.80)$ & $138.05(135.72-139.78)$ \\
Target dose (Gy) & $D 80$ & $170.76(160.21-178.47)$ & $165.12(160.02-177.79)$ \\
& $D 50$ & $276.57(254.96-322.01)$ & $262.33(245.89-319.85)$ \\
& $D 30^{*}$ & $394.30(362.31-431.00)$ & $361.06(339.73-400.54)$ \\
& V5 & $46.00(27.08-66.41)$ & $43.04(29.54-63.09)$ \\
All lung (\%) & V20 & $13.80(7.86-26.94)$ & $11.03(8.29-21.48)$ \\
& V30 & $8.07(4.95-16.67)$ & $7.69(5.30-15.10)$ \\
& MLD & $12.01(6.15-18.92)$ & $10.73(6.92-15.70)$ \\
Ipsilateral lung (\%) & V5 & $73.64(57.41-95.70)$ & $70.63(59.86-91.70)$ \\
& V20 & $27.74(18.05-57.33)$ & $23.91(17.09-47.94)$ \\
Heart (Gy) & V30 & $18.84(10.54-36.14)$ & $13.04(11.78-29.55)$ \\
Spinal cord (Gy) & MLD & $20.76(13.77-35.45)$ & $16.91(14.24-29.71)$ \\
Ribs (Gy) & D50 & $4.11(3.06-8.63)$ & $3.63(3.08-8.17)$ \\
\hline
\end{tabular}

VRBP: virtual single needle center point interstitial brachytherapy plan; ‘ ': $P<0.05$.

\section{Discussion}

In interstitial brachytherapy, after needles were implanted, dwell point position and dwell time were the principal methods of dose distribution optimization for planning [2-8]. In generally, more implantation needles could provide more dwell point position, this may in favor of the planning.

One of our results showed that the differences of lung dose were statistically significant between regular arrangement and irregular arrangement. It indicated that the effect of multiply needles arrangement on dose distribution do certainly exist and it might not be completely compensated by using the two optimization methods in 3D planning system. Thus, the arrangement of multiply needles was important and perhaps even a sufficient precondition.

The other results was that the differences of lung dose were not statistically significant between VSBP and AIBP. It indicated that the lung dose in interstitial brachytherapy plans with multiply needles irregular arrangement might have no obvious advantage over the VSBP. Although the high dose volume in interstitial brachytherapy plans with multiply needles irregular arrangement were smaller ( the $P$ value of D30 <0.05); more implantation needles prolong operation time and it might be accompanied with higher operation risk and more damage for patient. Thus, if multiple needles could not be arranged regularly, the statement of " more dwell point position may in favor of the planning" might be a "false proposition". Was it necessary to insert multiple needles should be worth considering.
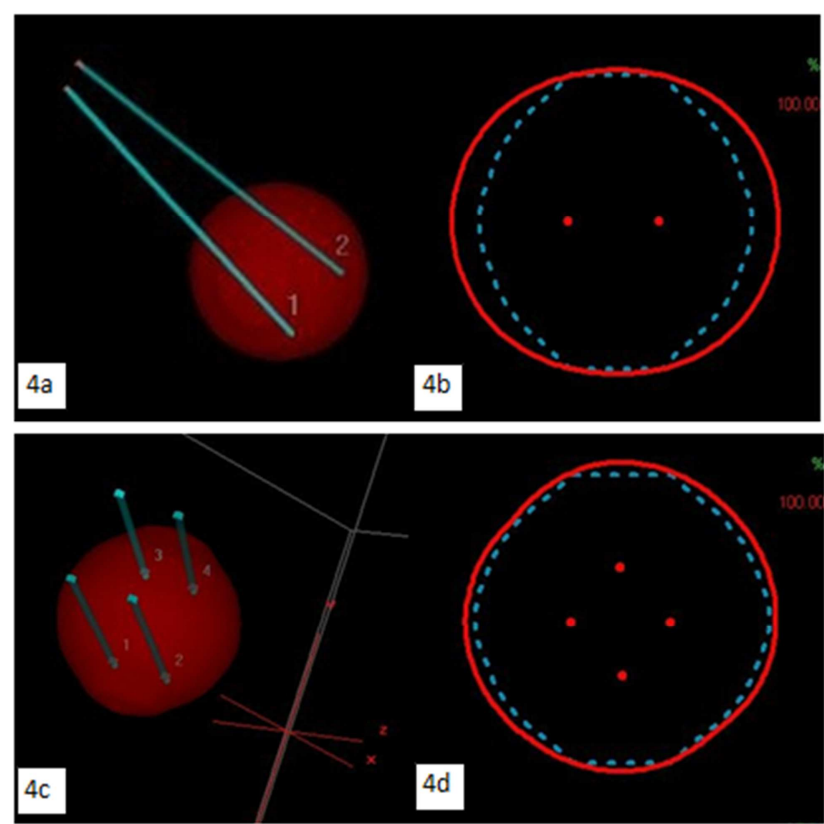

Figure 4. Schematic diagram of a spherical CTV for AIBP and VRBP the red solid line was the $100 \%$ prescription isodose curve, the dotted sky-bule line was CTV contour, the red dots were dwell points, The region encircled between the red solid line and the sky-blue dotted line indicated the area of the lung received exceeding 100\% prescription dose. $4 a$ : $3 D$ image for two needles with a $20^{\circ}$ angle; $4 \mathrm{~b}$ : dose distribution for 2 needles with a $20^{\circ}$ angle in a transverse section; $4 c$ : $3 D$ image for four parallel implantation needles with equal spacing; $4 d$ : dose distribution for four parallel implantation needles with equal spacing. 
For seeking the possible reason, we assumed that the CTV was a standard spherical volume. If two implantation needles with a $20^{\circ}$ angle were implanted into the spherical target volume. From the view of the insertion direction, two dwell points should be laid in a transverse section (Figure 4a). Due to the concentric circles of dose distribution surrounding each dwell point [2], the $100 \%$ prescription isodose curve could be an ellipse. If the $100 \%$ prescription isodose curve exactly covered all target areas, some of lung volume could be covered by this prescription isodose curve (Figure $4 b$ ).

If four parallel implantation needles with equal spacing were implanted into the spherical tCTV (Figure 4c). The mount of lung volume covered by this $100 \%$ prescription isodose curve might be less than it in two implantation needles plan (Figure 4d). This may be the reason why the lung dose in interstitial brachytherapy plans with irregular arrangement multiply needles higher than it in brachytherapy plans with regular arrangement.

Particularly, if one implantation needle was implanted and one dwell point was laid in the CTV center, the mount of lung volume covered by this $100 \%$ prescription isodose curve should be zero (Figure $5 \mathrm{a}$ and $5 \mathrm{~b}$ ). However, If the CTV was not a standard sphere, for example, it had a "defect"; some lung area was inevitably covered by $100 \%$ prescribed isodose carve and It was hard to alter (Figure 5c) due to the only one available dwell point. Conversely, the mount of lung volume covered by this $100 \%$ prescription isodose could be reduced by adjusting the time of the dwell point near the "defect" in multiply needles plans (AIBP and VRBP). In clinical, most of the clinical target volume were not a standard sphere, therefore, it produced the similar results of lung dose between VSBP and AIBP.
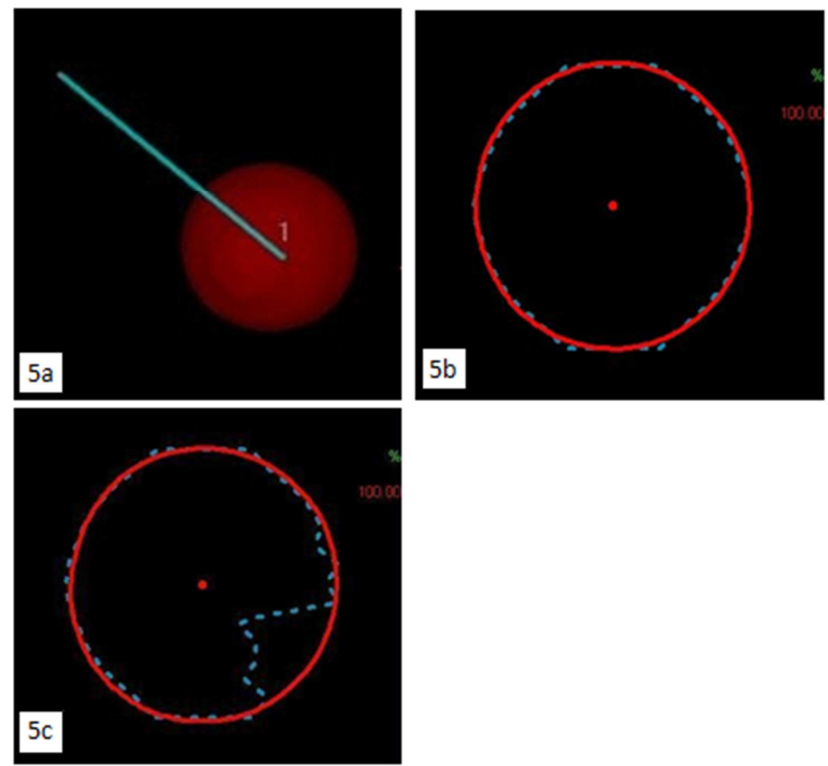

Figure 5. Schematic diagram of a spherical CTV for VSBP 5a: $3 D$ image for one needle passing through the CTV center. 5b: dose distribution for one needle, the CTV contour and the 100\% prescribed isodose curves were coincidence; $5 c$ : the dose distribution for the CTV with a "defect".

In relevant literature [9-17], there were lack of the description about needles alignment and studies of the effects on the dose distribution as well. Due to the interference of ribs and respiratory movement, a parallel arrangement of the multiple needles for lung tumor still has technical obstacles. Tselis et al. [12] had pointed out that a parallel needles alignment without course intersections inside the implanted volume was ideal. They had presented similar approach to VSBP for the spherical target with diameter less than $3 \mathrm{~cm}$ (volume: $\sim 14.13 \mathrm{~cm}^{3}$ ). We thought it might be also suitable for the volume no more than $150.13 \mathrm{~cm}^{3}$.

It is necessary to point out that this study was a research of hypothesis. The implantation needles in VRBP and VSBP were not actual. Due to ribs interference, multiply needles with regular arrangement was difficult to carry out in clinical. Perhaps, with help of bone drill the regular arrangement multiply needles might come true. Methods of utilizing the bone drill and the compromise between ribs damage caused by bone drill and lung dose should be further exploratory developed. However, we thought that there might have a chance of successful implementation for VSBP if the path of needle was carefully devised. In addition, respiratory motion may be a major obstacle to the implementation of this method, further research was needed to explore a technique for achieving VSBP.

The inclusion criteria (the CTV shape was approximately spherical and irregular arrangement of multiply needles) were important to our research, cases without meeting the inclusion criteria may not get the same results. The reason to adopt the large value $120 \mathrm{~Gy}$ as the prescription dose was in order to obtain reasonable metric data (For example, if 20 Gy was adopted as the prescription dose, the value of V30 were zero in all plans). One of limitations in our study was the limited sample size, further study with a larger sample size might yield more accurate results. Nevertheless, this results provided a valuable reference of interstitial brachytherapy for lung tumor.

\section{Conclusion}

In interstitial brachytherapy for lung tumor, the arrangement of implantation needles could influenced the dose distribution of lungs. If the CTV shape was approximately spherical and a regular arrangement of multiply needles was difficult to achieve; the lung dose of the AIBP might have no obvious advantage over the VSBP and the VSBP should be worth a try.

\section{Conflict of Interest}

The authors declare they have no conflicts of interest.

\section{Acknowledgements}

Thanks for Chinese Medical Association of Sichuan (Youth Innovation Project: Q15020) and Southwest Medical University Research Foundation (2016-129). 


\section{References}

[1] Shrieve D C. Basic principles of radiobiology applied to radiotherapy of benign intracranial tumors. [J]. Neurosurgery Clinics of North America, 2004, 15 (4): 467-79.

[2] Das, R. K. Icru 58 (dose and volume specification for reporting interstitial therapy), by international commission on radiation units and measurements. Medical Physics 2008; 25 (7), $1225-1225$.

[3] Kolkman I. Optimization of interstitial volume implants Radiotherapy and Oncology [J]. Radiotherapy \& Oncology Journal of the European Society for Therapeutic Radiology \& Oncology, 1994, 31 (3): 229-239.

[4] Bo Y, Xiaoyang S, Haowen P, et al. Comparative Study on Dose Distribution of Two Dwell Position Optimization Schemes in Interstitial Brachytherapy for Lung Cancer [J]. China Medical Devices, 2018, 33 (12): 57-60.

[5] Jamema S V, Saju S, Shetty U M, et al. Dosimetric comparison of inverse optimization with geometric optimization in combination with graphical optimization for HDR prostate implants.[J]. Journal of Medical Physics, 2006, 31 (2): 89-94.

[6] Anacak Y, Esassolak M, Aydin A, et al. Effect of geometrical optimization on the treatment volumes and the dose homogeneity of biplane interstitial brachytherapy implants [J]. Radiotherapy \& Oncology, 1997, 45 (1): 71-76.

[7] Rembowska A M E, Cook M, Hoskin P J, et al. The stepping source dosimetry system as an extension of the manchester system [J]. Radiotherapy \& Oncology, 1996, 39 (39): 25-25.

[8] Bo Y, Xiaoyang S, Haowen P, et al. Dosimetric analysis of rib interference of the CTV during interstitial brachytherapy of lung tumors [J]. Journal of Contemporary Brachytherapy, 2017, 9 (6): $566-571$.

[9] Ricke J, Wust P, Wieners G, et al. CT-guided interstitial single-fraction brachytherapy of lung tumors: phase I results of a novel technique. [J]. Chest, 2005, 127 (6): 2237.

[10] Imamura F, Ueno K, Kusunoki $\mathrm{Y}$, et al. High-dose-rate brachytherapy for small-sized peripherally located lung cancer [J]. Strahlentherapie und Onkologie, 2006, 182 (12): 703-707.

[11] Sharma D N, Rath G K, Thulkar S, et al. Computerized tomography-guided percutaneous high-dose-rate interstitial brachytherapy for malignant lung lesions. [J]. Journal of Cancer Research \& Therapeutics, 2011, 7 (2): 174-179.

[12] Peters N, Wieners G, Pech M, et al. CT-guided interstitial brachytherapy of primary and secondary lung malignancies: results of a prospective phase II trial [J]. Strahlentherapie und Onkologie, 2008, 184 (6): 296.

[13] Stewart A, Parashar B, Patel M, et al. American Brachytherapy Society consensus guidelines for thoracic brachytherapy for lung cancer [J]. Brachytherapy, 2015, 15 (1): 1.

[14] Lee A, Supariwala A, Venkat P, et al. Interstitial CT-Guided Lung Brachytherapy [J]. Brachytherapy, 2019, 18 (3): S84-S85.

[15] Dou H, Jiang S, Yang Z, et al. Design and validation of a CT-guided robotic system for lung cancer brachytherapy [J]. Medical Physics, 2017, 44 (9).

[16] Irina F, Kyveli Z, Vasileios L, et al. A comparative assessment of inhomogeneity and finite patient dimension effects in, $60 \mathrm{Co}$, and, $192 \mathrm{Ir}$ high-dose-rate brachytherapy [J]. Journal of Contemporary Brachytherapy, 2018, 10 (1): 73-84.

[17] Tselis N, Ferentinos K, Kolotas C, et al. Computed tomography-guided interstitial high-dose-rate brachytherapy in the local treatment of primary and secondary intrathoracic malignancies $[\mathrm{J}]$. Journal of Thoracic Oncology Official Publication of the International Association for the Study of Lung Cancer, 2011, 6 (3): 545. 